\title{
Mott transition in granular aluminum
}

\author{
N. Bachar, ${ }^{1, *}$ S. Lerer, ${ }^{1}$ A. Levy, ${ }^{1}$ S. Hacohen-Gourgy,,${ }^{1}$ B. Almog, ${ }^{1}$ H. Saadaoui, ${ }^{2}$ Z. Salman, ${ }^{2}$ \\ E. Morenzoni, ${ }^{2}$ and G. Deutscher ${ }^{1}$ \\ ${ }^{1}$ Raymond and Beverly Sackler School of Physics and Astronomy, Tel Aviv University, Tel Aviv, 69978, Israel \\ ${ }^{2}$ Laboratory for Muon Spin Spectroscopy, Paul Scherrer Institute, 5232 Villigen PSI, Switzerland \\ (Received 18 August 2014; revised manuscript received 29 December 2014; published 29 January 2015)
}

\begin{abstract}
A Mott transition in granular Al films is observed by probing the increase of the spin-flip scattering rate of conduction electrons as the nanosize metallic grains are being progressively decoupled. The presence of free spins in granular Al films is directly demonstrated by $\mu \mathrm{SR}$ measurements. Analysis of the magnetoresistance in terms of an effective Fermi energy shows that it becomes of the order of the grains electrostatic charging energy at a room temperature resistivity $\rho_{300 \mathrm{~K}} \approx 50000 \mu \Omega \mathrm{cm}$, at which a metal to insulator transition is known to exist. As this transition is approached the magnetoresistance exhibits a heavy-fermion-like behavior, consistent with an increased electron effective mass.
\end{abstract}

DOI: 10.1103/PhysRevB.91.041123

PACS number(s): 74.81.Bd, 71.30.+h, 72.15.Qm, 74.25.Ha

Thanks to advances in the development of the dynamical mean field theory (DMFT) [1], considerable progress has been made in recent years towards a detailed understanding of the Mott metal to insulator transition, predicted to occur when the electron-electron interaction is of the order of the bandwidth [2]. However, the experimental observation of this transition has remained a challenge in three-dimensional systems. This is because in a homogeneous metal the Coulomb interaction is smaller by several orders of magnitude than the bandwidth, even in the presence of a relatively high concentration of impurities [3,4].

We show here that a Mott transition takes place in granular metals, as nanosize grains are decoupled from each other by a progressive reduction of the intergrain tunneling probability. Two of the main features of this transition predicted by DMFT theory, an increase of the electron effective mass and a noncritical behavior of the electronic density of states as the transition is approached, have been observed.

These observations have been made possible by the presence of free spins in granular aluminum films, which we confirm here directly by $\mu \mathrm{SR}$ measurements. Interaction of these spins with conduction electrons results in a negative magnetoresistance [5]. We have used it as a tool to follow changes of the effective Fermi energy of the granular medium as the transition is being approached. When it occurs, at a room temperature resistivity of about $50000 \mu \Omega \mathrm{cm}$, we find that the effective Fermi energy is of the order of the grain's charging electrostatic energy, showing that it is of the Mott type.

In a previous publication [6] we showed that the resistance of granular Al films exhibit a Kondo-like behavior with a minimum followed by a $\ln (T)$ dependence at low temperatures. Their magnetoresistance is negative. At high temperatures and low magnetic fields the negative magnetoresistance scales as $(H / T)^{n}$ with $n$ close to 2 . This is an indirect evidence for a spin-flip scattering mechanism between the conduction electrons and localized magnetic moments [5]. We show in this Rapid Communication a direct observation of these localized moments by $\mu$ SR measurements. Moreover, the low field $H^{2}$

\footnotetext{
*nimrodb7@post.tau.ac.il
}

dependence is predicted to change to a $\ln H$ dependence at high magnetic fields [7]. At temperatures on the order of $T_{c}$ and below and at high magnetic fields on the order of $20 \mathrm{~T}$, we observe as expected a sign change of $d^{2} \rho / d H^{2}$ towards saturation at higher magnetic fields.

Low energy muon spin rotation/relaxation (LE- $\mu$ SR) experiments [8] were performed on films with resistivities of approximately 140, 300, 1200, 1800, and $10000 \mu \Omega$. The measurements were performed at the Swiss Muon Source on the $\mu \mathrm{E} 4$ beamline, at the Paul Scherrer Institute, in Switzerland. With implantation energy of $10 \mathrm{keV}$ all the muons stop in the $100 \mathrm{~nm}$ film, with a mean range of $68 \mathrm{~nm}$ and a stopping width $(\mathrm{rms})$ of $15 \mathrm{~nm}$. The time evolution of the polarization of the muon ensemble implanted in the samples, $P(t)$, measured via detection of the emitted decay positron intensity as a function of time after thermalization, is very sensitive to the local magnetic environment and to the presence of spins. Polarization spectra taken at different temperatures under zero field conditions decay with a small rate, which has two contributions. The first, which is temperature independent, is due to the nuclear moments of Al. In addition a temperature dependent exponential relaxation of electronic origin is observable with rate $\lambda$ (typical behavior is shown in Fig. 1 for a sample at low resistivities). This rate reaches a value of $\lambda=0.085 \mu \mathrm{s}^{-1}$ at a temperature corresponding to the low temperature increase of the resistivity. The analysis of muon spin rotation measurements on the entire set of films shows a relaxation rate of $\lambda=0.09 \pm 0.01 \mu \mathrm{s}^{-1}$ corresponding to a concentration level of $400 \pm 50 \mathrm{ppm}$. More information about the $\mu \mathrm{SR}$ data and their analysis is provided in the Supplemental Material [9].

The presence of localized magnetic moments in bulk $\mathrm{Al}$ is not obvious. However, magnetic properties are expected in small metallic particles due to electron confinement [10] that may lead to odd-even Kondo nanodot behavior [11]. Alternately, scattering can occur with spins located at the metal-oxide interface between neighboring grains. Flux noise and other magnetometric measurements on $\mathrm{Al}$ oxide interface resulted in a surface spin density of approximately $5 \times$ $10^{17} \mathrm{~m}^{-2}$ [12-14]. A surface spin density of approximately $10^{16} \mathrm{~m}^{-2}$ was shown to fit the $1 / f$ flux noise observed in superconducting devices by Faoro and Ioffe [15]. Considering 


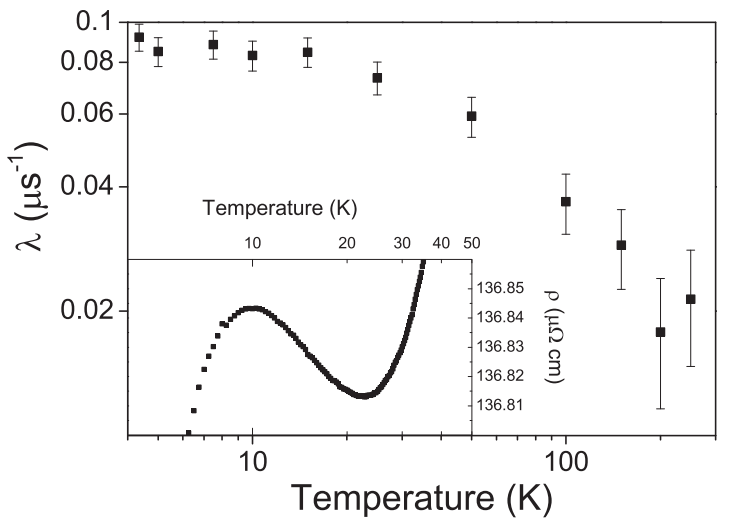

FIG. 1. Temperature dependence of the muon spin relaxation rate of electronic origin $\lambda$ for a sample with $\rho_{300 \mathrm{~K}} \approx 140 \mu \Omega \mathrm{cm}$. $\lambda$ appears to saturate around a temperature where the resistivity starts to increase [6] (inset).

that our grains have a size of about $2 \mathrm{~nm}$, we calculate a spin interface density of about $10^{16} \mathrm{~m}^{-2}$.

The fabrication of granular $\mathrm{Al}$ films has been described elsewhere in detail [16]. Briefly, Al is evaporated in the presence of a reduced pressure of oxygen. The films were deposited onto a substrate, held either at room temperature or at liquid nitrogen temperature. They consist of nanoscale grains of crystalized aluminum separated by thin oxide barriers. All samples studied had a thickness of $100 \mathrm{~nm}$. The respective amounts of $\mathrm{Al}$ metallic grains and $\mathrm{Al}$ oxide were controlled by the $\mathrm{Al}$ evaporation rate and oxygen partial pressure during evaporation. As the oxide volume fraction is increased the grain size first goes down and eventually reaches a constant value of about $2 \mathrm{~nm}$ for films deposited at liquid nitrogen temperature, and $3 \mathrm{~nm}$ for films deposited at room temperature. This occurs when the room temperature value of the macroscopic resistivity reaches about $100 \mu \Omega \mathrm{cm}$. As the macroscopic resistivity increases, $T_{c}$ rises from that of bulk $\mathrm{Al}(1.2 \mathrm{~K})$, reaches a rather flat maximum value of $3.2 \mathrm{~K}$ (films deposited at liquid nitrogen temperature) or $2.2 \mathrm{~K}$ (films deposited at room temperature) for resistivity values ranging from 100 to $300 \mu \Omega \mathrm{cm}$, before slowly going down for resistivities higher than $500 \mu \Omega \mathrm{cm}$. A film deposited at liquid nitrogen temperature having a resistivity of about $10000 \mu \Omega \mathrm{cm}$ is still superconducting at $1.8 \mathrm{~K}$.

The negative magnetoresistance of a metal containing magnetic impurities is due, at low fields, to the difference in the scattering rates of spin up and spin down electrons. In the presence of a magnetic field oriented along the positive $z$ direction there are more scattering events of a spin down electron into a spin up than that of a spin up electron scattering into a spin down. But the lower density of available states below the Fermi level where spin up electrons end up due to the Zeeman energy gain forbids these scattering processes because of the exclusion principle [5]. The total contribution of the spin-flip scattering amplitudes to the resistivity is decreased. The difference in the scattering rates of the two processes increases as $\alpha^{2}=\left(g \mu_{B} H / k_{B} T\right)^{2}$, and so does the magnetoresistance. In this regime the field dependence of the scattering amplitudes is negligible. The interpretation of the $(H / T)^{2}$ dependence of the negative magnetoresistance of our films in terms of the presence of localized magnetic moments, proposed in a previous publication [6], is confirmed by the present direct observation of free spins.

Here we focus on the large increase of the magnetoresistance seen as $\mathrm{Al}$ grains are being progressively decoupled and a metal to insulator transition is approached. In dilute alloys the magnetoresistance is proportional to the magnetic impurity concentration [5]. Since the magnetic impurity concentration determined by $\mu \mathrm{SR}$ is about the same for all films studied, one would expect their magnetoresistance to be about the same. However, it increases by several orders of magnitude [6] rising in fact faster than the resistivity itself with a power law $\Delta \rho \propto \rho^{1.38}$. The origin of the increasing spin scattering of conduction electrons must lie, we believe, in an increase of the scattering amplitudes as the metal to insulator transition is approached.

Since the magnetoresistance results from the difference of the scattering rates of spin up and spin down electrons, we can write it as

$$
\Delta \rho=\rho_{+}-\rho_{-}=\frac{m^{*}}{n e^{2}}\left(\frac{1}{\tau_{+}}-\frac{1}{\tau_{-}}\right) .
$$

The scattering rates are given as

$$
\frac{1}{\tau_{ \pm}}=\frac{k m^{*} v_{0} c}{\pi \hbar^{3}} f\left(V, J,\left\langle S_{z}\right\rangle,\left\langle S_{z}^{2}\right\rangle, S, \epsilon_{ \pm}, \alpha\right),
$$

where $k$ and $m^{*}$ are, respectively, the wave number and effective mass of the conduction electron, $v_{0}$ is the atomic volume of the host metal, and $c$ is the magnetic impurity concentration in ppm. $f$ is a general function defined in Ref. [5] by the Coulomb interaction $V$, the interaction constant $J$, the equilibrium average of the operators $S_{z}$ and $S_{z}^{2}$ for the spin component in the $z$ direction, the impurity spin $S$, the energy shifts $\epsilon_{ \pm}=E_{F} \mp \mu_{B} H$ of the spin up/down final states, and $\alpha=\left(g \mu_{B} H / k_{B} T\right)$ with the $g$ value of the impurity.

We assume that the large increase of the magnetoresistance is primarily due to variations of the carrier density $n$ and of the effective mass $m^{*}$ as grains are being progressively decoupled:

$$
\Delta \rho \propto \frac{m^{*^{2}}}{n} .
$$

To evaluate the increase of the effective mass with the normal state resistivity, we use the Hall mobility result [17]

$$
\mu_{H} \propto \rho^{-0.5} \text {. }
$$

Bandyopadhyay et al. have presented their results as a function of the normal state resistivity measured at $4.2 \mathrm{~K}$, but for samples for which $\rho<2 \times 10^{4} \mu \Omega \mathrm{cm}$ the temperature dependence of the resistivity is negligible and the Hall carrier concentration varies as a function of $\rho$ in the same way as the mobility does. Since the magnetoresistance varies as $\rho^{1.38}$, we conclude that

$$
m^{*} \propto \rho^{0.44}
$$

For the cleaner sample measured by Bandyopadhyay et al., with $\rho \approx 230 \mu \Omega \mathrm{cm}$, the value of the Hall constant is $R_{H}=$ $\mu_{H} \cdot \rho=0.47 \times 10^{-10} \Omega \mathrm{m} / \mathrm{T}$, getting close to the bulk Al value of $0.3 \times 10^{-10} \Omega \mathrm{m} / \mathrm{T}$. Therefore, we will assume that 


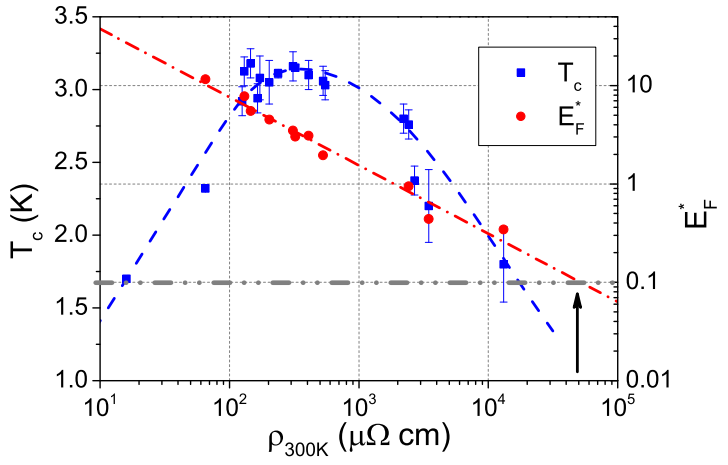

FIG. 2. (Color online) Critical temperature (blue squares) $T_{c}$ and effective Fermi energy (red dots) as a function of the room temperature resistivity. The dashed line is a guide to the eye, the dash dotted line follows $E_{F}^{*} \propto \rho^{-0.7}$ and the dash double dotted line is showing the limit of the electrostatic energy for a $2 \mathrm{~nm}$ grain. The arrow marker points to the critical resistivity $\rho \approx 50000 \mu \Omega \mathrm{cm}$ at which a Mott transition should occur.

in samples having normal state resistivities lower than $\rho \approx$ $100 \mu \Omega \mathrm{cm}$ the basic electronic properties-effective mass and Fermi energy-have their bulk values. This assumption is also in agreement with the fact that the upper critical field $B_{c 2}(0)$ of granular $\mathrm{Al}$ films is proportional to the resistivity $\rho$ up to $100 \mu \Omega \mathrm{cm}$ [18]. This proportionality is in agreement with the behavior of a conventional type II superconductor. This proportionality is not observed beyond $100 \mu \Omega \mathrm{cm}$ [19] implying that electronic properties such as the density of states, the Fermi velocity, and the effective Fermi energy are modified. According to Eq. (5), the electron effective mass increases by a factor 10 in sample having resistivity of $\rho \approx 10000 \mu \Omega \mathrm{cm}$.

In order to discuss the approach to the metal to insulator transition it is convenient to present Eq. (26) of Ref. [5] in a slightly different form:

$$
\Delta \rho=-\beta \frac{\hbar}{e^{2}} a c_{i}\left(\frac{J}{E_{F}}\right)^{2} \alpha^{2} u,
$$

where $\beta$ is a number that depends on the atomic packing factor of the material, $\hbar / e^{2}$ is the quantum resistance, $a$ is the interatomic distance, $c_{i}$ is the magnetic impurity concentration in ppm, $J$ is the interaction constant, $E_{F}$ is the Fermi energy, and $\alpha$ and $u$ are a function of the spin of the impurity $S$, the Coulomb interaction $V, J$, and $E_{F}$. Figure 2 shows the variation of the effective Fermi energy as a function of the room temperature resistivity of the films, assuming that all other factors in Eq. (6) remain constant, $T=20 \mathrm{~K}, H=14 \mathrm{~T}$, and an impurity concentration of $400 \mathrm{ppm}$. In order to evaluate the unknown factor $\beta J^{2} u$ we have assumed that the value of the Fermi energy in the lowest resistivity sample showing the magnetoresistance scaling behavior $(\rho \approx 65 \mu \Omega \mathrm{cm}$ ) is that of the bulk $E_{F}=11.6 \mathrm{eV}$. This is in keeping with the fact that at this resistivity the Hall constant is unchanged from the bulk value, as noted above.

As can be seen from Fig. 2, $E_{F} \propto \rho^{-0.7}$. Its extrapolated value hits the electrostatic charging energy $U=85 \mathrm{meV}$ of the grains at a room temperature resistivity of about $50000 \mu \Omega \mathrm{cm}$, the value of $U$ being calculated using the grain size of $2 \mathrm{~nm}$ and the dielectric constant of $\mathrm{Al}_{2} \mathrm{O}_{3}, \varepsilon=8.5$.
An accepted criterion for the resistivity $\rho_{300 \mathrm{~K}}$ at which the metal to insulator transition occurs is given by the value where the low temperature dependence of the resistivity becomes exponential. For films deposited at liquid nitrogen temperature having a grain size of $2 \mathrm{~nm}$, a metal like $(d \rho / d T>0)$ to insulator like $(d \rho / d T<0)$ transition has been observed but with $\ln (T)$ dependence rather than an exponential one, therefore all samples are in the metallic side [6]. For films deposited at room temperature having a larger grain size (3 $\mathrm{nm}$ ) than in our films, measurements performed down to $1.2 \mathrm{~K}$ gave a critical resistivity for the exponential criterion a value between 20000 and $50000 \mu \Omega \mathrm{cm}$ [20]. However, measurements performed down to $0.06 \mathrm{~K}$ gave values higher than $20,000 \mu \Omega \mathrm{cm}[21,22]$. These values are definitely higher than the value of $50000 \mu \Omega \mathrm{cm}$ at which the effective $E_{F}^{*}$ is down to the charging energy of $2 \mathrm{~nm}$ grain size samples. This is in accordance with the smaller charging energy of the $3 \mathrm{~nm}$ grain.

For the sake of comparison with the results of previous works obtained on $3 \mathrm{~nm}$ grain size samples we have made a few checks on magnetoresistance $(\Delta \rho)$ of such films. No major change was observed; at $20 \mathrm{~K}$ and $14 \mathrm{~T}$, a $110 \mu \Omega \mathrm{cm}$ film showed $\Delta \rho \approx 0.07 \mu \Omega \mathrm{cm}$; a $1355 \mu \Omega \mathrm{cm}$ film showed $\Delta \rho \approx 3 \mu \Omega \mathrm{cm}$. These data points fall on the same power law line $\Delta \rho \propto \rho^{1.38}$ as found for the $2 \mathrm{~nm}$ grain size samples. A full comparison with larger grains is beyond the scope of the present paper and will be the subject of further work.

The metal to insulator transition in nanoscale granular aluminum occurs when the effective Fermi energy becomes of the order of the electrostatic charging energy of the grains. This strongly suggests that the transition is of the Mott type. Since the density of states varies as $n / E_{F}$ and since $n \propto \rho^{-0.5}$, the dependence of the density of states on $\rho$ is quite weak, $N(0) \propto \rho^{0.2}$. This is consistent with the predicted noncritical behavior of the density of states at the Mott transition [1].

Kawabata has calculated the bandwidth of a system consisting of small metallic particles with an electronic level splitting $\delta$ and intergrain electron tunneling matrix element $t$. Assuming that there are $z$ adjacent particles, he finds in the weak coupling limit a bandwidth $w \cong \pi z t^{2} / \delta$ [23]. Since the grain size and therefore $\delta$ should be roughly constant, the control parameter is $t$, which varies with the resistivity as $\rho^{-1} \propto t^{2}$ [24]. As the grain's oxide barrier thickness increases, so does the resistivity, $t$ reduces and as a result $w$ decreases. However, these calculations do not take into account electron-electron interactions.

The increasing effective mass in high resistivity films might induce some similarities with the case of heavy fermions. Such similarity is indeed observed in their respective magnetoresistance behaviors. In the Kondo diluted case the magnetoresistance remains negative at all temperatures. But in heavy fermions there is a change of sign of the magnetoresistance at low temperatures at a fixed field $[25,26]$. In the most resistive $\left(\rho_{300 \mathrm{~K}} \approx 13000 \mu \Omega \mathrm{cm}\right)$ superconducting sample measured, which still has a critical temperature of $1.8 \mathrm{~K}$, the low field magnetoresistance turns positive below $20 \mathrm{~K}$, as can be seen in Fig. 3. Down to $T=8 \mathrm{~K}$, the field at which the magnetoresistance changes sign, remains of the order of $2 \mathrm{~T}$. It is only below $8 \mathrm{~K}$ that the magnetoresistance changes sign at a higher field, due to superconducting fluctuations 


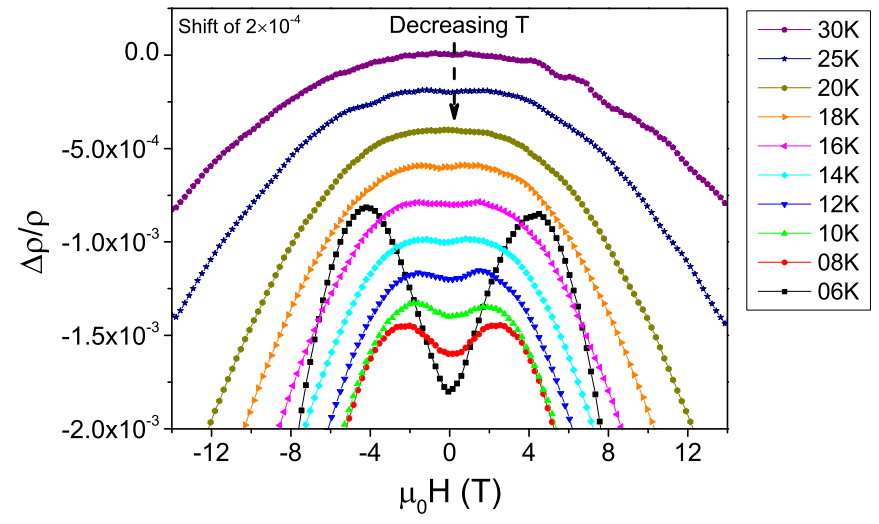

FIG. 3. (Color online) Magnetoresistance of a high resistivity film $(\rho \approx 13000 \mu \Omega \mathrm{cm})$ at low temperatures. Below $20 \mathrm{~K}$, the magnetoresistance is positive at low fields and negative at high fields. Down to $8 \mathrm{~K}$ the variation of the magnetoresistance changes sign at a fixed field, as is the case for a Kondo lattice.

known as the ghost critical field (GCF) effect [27]. Therefore, above $8 \mathrm{~K}$, the change of sign in the magnetoresistance is a property of the normal state where granular $\mathrm{Al}$ films show a heavy-fermion-like behavior.

Finally, we would like to address the effect of spin-flip scattering on $T_{c}$. In a perturbed BCS superconductor $T_{c}$ decreases as $[28,29]$

$$
\ln \left(\frac{T_{c}}{T_{c 0}}\right)=\psi\left(\frac{1}{2}\right)-\psi\left(\frac{1}{2}+\frac{\hbar}{4 \pi k_{B} T_{c} \tau_{s}}\right),
$$

where $T_{c 0}$ is the critical temperature of an unperturbed superconductor, $\psi(x)$ is the digamma function, and $\hbar / \tau_{s}$ is given by [30]

$$
\frac{\hbar}{\tau_{s}}=\frac{\pi}{4} c_{i} S(S+1) N(0)|J|^{2} .
$$

We have established above that the resistivity dependence of the density of states is negligible. Considering $N(0)=$ $0.381 \mathrm{eV}^{-1}$ for aluminum, typical values of $S=0.5, J=$ $1 \mathrm{eV}$, and a concentration of $400 \mathrm{ppm}$, we get $\hbar / \tau_{s} \approx 0.1 \mathrm{meV}$. This gives $T_{c} \approx 2.73 \mathrm{~K}$ for a superconductor with $T_{c 0} \approx 3.2 \mathrm{~K}$. Therefore, the measured spin concentration is insufficient in order to explain the decrease of $T_{c}$ as the metal to insulator transition is approached.

A more probable explanation of the decrease of $T_{c}$ lies in the reduced Fermi energy. For the sample with $\rho_{300 K} \approx$ $13000 \mu \Omega \mathrm{cm}$ (Fig. 3), the Fermi energy is down to $0.34 \mathrm{eV}$. We then calculate an effective Coulomb pseudopotential $\mu^{*} \approx$ 0.15 instead of the standard value of $\mu^{*} \approx 0.1$ [31]. This leads to a reduced $T_{c}$ of about $1 \mathrm{~K}$ even smaller than the measured $T_{c}[31]$.

In conclusion, the scattering of conduction electrons by free spins, whose presence in granular $\mathrm{Al} / \mathrm{Al}_{2} \mathrm{O}_{3}$ films was directly confirmed by muon spin rotation experiments, increases by several orders of magnitude as the grains are progressively decoupled. This large increase is attributed to the decrease in the effective Fermi energy. At high resistivities it approaches the electrostatic energy of the grain and a Mott transition is likely to occur. The critical resistivity at this transition is in agreement with previous experimental observations of a metal to insulator transition in granular Al films. A heavyfermion-like behavior is observed in the magnetoresistance of the films as the Mott transition is approached and the effective mass increases. Elucidation of the coexistence of free spins in granular $\mathrm{Al}$ with enhanced superconductivity requires further work.

Useful discussions with Philippe Nozières are gratefully acknowledged. The $\mu \mathrm{SR}$ measurements were performed at the Swiss Muon Source $(\mathrm{S} \mu \mathrm{S})$, Paul Scherrer Institute, Switzerland. N.B. and A.L. acknowledge travel support within the Access Programm of FP7. This work was partially supported by EOARD Award FA8655-10-1-3011.
[1] A. Georges, G. Kotliar, W. Krauth, and M. J. Rozenberg, Dynamical mean-field theory of strongly correlated fermion systems and the limit of infinite dimensions, Rev. Mod. Phys. 68, 13 (1996).

[2] N. F. Mott, The basis of the electron theory of metals, with special reference to the transition metals, Proc. Phys. Soc. Sect. A 62, 416 (1949); Metal-insulator transition, Rev. Mod. Phys. 40, 677 (1968).

[3] N. F. Mott, Metal-Insulator Transitions, 2nd ed. (Taylor and Francis, London, 1974).

[4] M. Imada, A. Fujimori, and Y. Tokura, Metal-insulator transitions, Rev. Mod. Phys. 70, 1039 (1998).

[5] M.-T. Béal-Monod and R. A. Weiner, Negative magnetoresistivity in dilute alloys, Phys. Rev. 170, 552 (1968).

[6] N. Bachar, S. Lerer, S. Hacohen-Gourgy, B. Almog, and G. Deutscher, Kondo-like behavior near the metal-to-insulator transition of nanoscale granular aluminum, Phys. Rev. B 87, 214512 (2013).

[7] R. Weiner and M. Béal-Monod, High-field behavior in the kondo effect, Phys. Rev. B 3, 145 (1971).
[8] E. Morenzoni, T. Prokscha, A. Suter, H. Luetkens, and R. Khasanov, Nano-scale thin film investigations with slow polarized muons, J. Phys.: Condens. Matter 16, S4583 (2004).

[9] See Supplemental Material at http://link.aps.org/supplemental/ 10.1103/PhysRevB.91.041123 for typical $\mu$ SR polarization spectra and detailed analysis of the spin concentration.

[10] R. Kubo, Electronic properties of metallic fine particles. I., J. Phys. Soc. Jpn. 17, 975 (1962).

[11] S. M. Cronenwett, T. H. Oosterkamp, and L. P. Kouwenhoven, A tunable kondo effect in quantum dots, Science 281, 540 (1998).

[12] S. Sendelbach, D. Hover, A. Kittel, M. Mück, J. M. Martinis, and R. McDermott, Magnetism in SQUIDs at millikelvin temperatures, Phys. Rev. Lett. 100, 227006 (2008).

[13] S. M. Anton, J. S. Birenbaum, S. R. O'Kelley, V. Bolkhovsky, D. A. Braje, G. Fitch, M. Neeley, G. C. Hilton, H.-M. Cho, K. D. Irwin, F. C. Wellstood, W. D. Oliver, A. Shnirman, and J. Clarke, Magnetic flux noise in dc SQUIDs: Temperature and geometry dependence, Phys. Rev. Lett. 110, 147002 (2013). 
[14] H. Bluhm, J. A. Bert, N. C. Koshnick, M. E. Huber, and K. A. Moler, Spinlike susceptibility of metallic and insulating thin films at low temperature, Phys. Rev. Lett. 103, 026805 (2009).

[15] L. Faoro and L. B. Ioffe, Microscopic origin of low-frequency flux noise in josephson circuits, Phys. Rev. Lett. 100, 227005 (2008).

[16] G. Deutscher, H. Fenichel, M. Gershenson, E. Grünbaum, and Z. Ovadyhu, Structural and superconducting properties of granular aluminum films, Low Temp. Phys.-LT 13 5, 573 (1974); G. Deutscher, H. Fenichel, M. Gershenson, E. Grünbaum, and Z. Ovadyahu, Transition to zero dimensionality in granular aluminum superconducting films, J. Low Temp. Phys. 10, 231 (1973); G. Deutscher, M. Gershenson, E. Grünbaum, and Y. Imry, Granular superconducting films, J. Vac. Sci. Technol. 10, 697 (1973).

[17] B. Bandyopadhyay, P. Lindenfeld, W. L. McLean, and H. K. Sin, Hall effect in granular aluminum, Phys. Rev. B 26, 3476(R) (1982).

[18] B. Abeles, R. Cohen, and W. Stowell, Critical magnetic fields of granular superconductors, Phys. Rev. Lett. 18, 902 (1967).

[19] T. Chui, P. Lindenfeld, W. L. McLean, and K. Mui, Coupling and isolation: Critical field and transition temperature of superconducting granular aluminum, Phys. Rev. B 24, 6728 (1981).

[20] G. Deutscher, B. Bandyopadhyay, T. Chui, P. Lindenfeld, W. L. McLean, and T. Worthington, Transition to localization in granular aluminum films, Phys. Rev. Lett. 44, 1150 (1980).

[21] M. Kunchur, P. Lindenfeld, W. L. McLean, and J. S. Brooks, Absence of superconductivity in metallic granular aluminum, Phys. Rev. Lett. 59, 1232 (1987).
[22] T. A. Miller, M. Kunchur, Y. Z. Zhang, P. Lindenfeld, and W. L. McLean, Superconductivity and the metal-insulator transition: Tuning with spin-orbit scattering, Phys. Rev. Lett. 61, 2717 (1988).

[23] A. Kawabata, Effects of tunneling on the properties of metallic fine particles, J. Phys. Soc. Jpn. 43, 1491 (1977).

[24] I. Beloborodov, a. Lopatin, V. Vinokur, and K. Efetov, Granular electronic systems, Rev. Mod. Phys. 79, 469 (2007).

[25] U. Rauchschwalbe, F. Steglich, A. de Visser, and J. Franse, Magnetoresistance of Ce-based Kondo lattices: $\mathrm{CeCu}_{2} \mathrm{Si}_{2}$ and $\mathrm{CeAl}_{3}$, J. Magn. Magn. Mater. 63-64, 347 (1987).

[26] L. Taillefer, J. Flouquet, and W. Joss, High-field magnetoresistance of $\mathrm{UPt}_{3}$, J. Magn. Magn. Mater. 76-77, 218 (1988).

[27] A. Kapitulnik, A. Palevski, and G. Deutscher, Inhomogeneity effects on the magnetoresistance and the ghost critical field above $\mathrm{T}_{\mathrm{c}}$ in thin mixture films of In-Ge, J. Phys. C: Solid State Phys. 18, 1305 (1985).

[28] A. A. Abrikosov and L. P. Gor'kov, Contribution to the theory of superconducting alloys with paramagnetic impurities, Zh. Eksp. Teor. Fiz. 39, 1781 (1960) [Sov. Phys. JETP 12, 1243 (1961)].

[29] K. Maki, Superconductivity (Marcel Dekker, New York, 1969), Chap. Gapless Superconductivity.

[30] A. Ludwig and M. J. Zuckermann, The dependence of the superconducting critical temperature of dilute magnetic alloys on impurity concentration, J. Phys. F: Metal Phys. 1, 516 (1971).

[31] P. Morel and P. Anderson, Calculation of the superconducting state parameters with retarded electron-phonon interaction, Phys. Rev. 125, 1263 (1962). 